\section{Avaliação da adequação das informações de mortalidade e nascidos vivos no Estado de Pernambuco, Brasil}

\author{
Evaluation of data on mortality and live births \\ in Pernambuco State, Brazil
}

\begin{abstract}
The Brazilian Ministry of Health has made important investments to improve the quality of information systems on mortality and live births. However, in most Brazilian States the infant mortality rate is still estimated by indirect methods, thus overlooking the progress in this area. The current article aimed to evaluate the adequacy of mortality and live birth data using indicators of coverage and regularity for both systems. Analysis of temporal trends in infant mortality coverage estimated by indirect methods revealed major inconsistencies in the temporal series. The adequacy indicators showed an important improvement in the coverage and regularity of vital information systems. The proportion of deaths from ill-defined causes also decreased, although at a slower rate. The improvement in recording of live births and deaths in the State of Pernambuco highlights the importance of reflecting on the use of direct methods to calculate infant mortality, at least in municipalities with adequate vital statistics.
\end{abstract}

Information Systems; Live Birth; Infant Mortality; Health Evaluation
Paulo Germano de Frias 1,2 Pricila Melissa Honorato Pereira 1 Carla Lourenço Tavares de Andrade 3 Pedro Israel Cabral de Lira 4 Célia Landmann Szwarcwald 5

\section{Introdução}

O Sistema de Informações sobre Nascidos Vivos (SINASC) e o Sistema de Informações sobre Mortalidade (SIM) são as fontes de informação do Ministério da Saúde que possibilitam o monitoramento dos eventos vitais no Brasil e permitem a construção de indicadores de saúde de forma contínua para todo o país.

O reconhecimento da importância de monitoramento das informações sobre óbitos e nascimentos junto à facilidade de acesso aos dados têm resultado no aumento substancial na cobertura e na qualidade das informações de ambos os sistemas 1. Entretanto, a persistente precariedade dos dados em alguns estados das regiões menos desenvolvidas do país (Norte e Nordeste) ainda não permite o cálculo direto da mortalidade infantil, em âmbito nacional, resultando na utilização de estimativas elaboradas pelo Instituto Brasileiro de Geografia e Estatística (IBGE). Tais estimativas são baseadas em dados dos censos populacionais e das Pesquisas Nacionais por Amostra de Domicílios (PNAD) e calculadas por técnicas demográficas indiretas de mensuração. Em apenas sete estados do Brasil e no Distrito Federal, recomenda-se o uso do método direto para o cálculo da mortalidade infantil 2.

A fidedignidade das estimativas indiretas, porém, depende do tamanho da amostra, do tipo de estudo e da forma como o inquérito foi conduzido. Geralmente, por conta do tamanho insu- 
ficiente da amostra, as técnicas de mensuração indireta não podem ser aplicadas no nível municipal, limitando o monitoramento da mortalidade infantil justamente nas áreas com os maiores problemas de saúde. Além disso, as estimativas são tratadas como parâmetros populacionais sem estimação dos erros de amostragem 3 .

Problemas nas estimativas obtidas por técnicas de mensuração indireta têm sido evidenciados, igualmente, em trabalhos nacionais. Estudo que comparou as taxas obtidas por cálculo direto com as estimadas por técnicas de mensuração indireta mostrou inconsistências relevantes nos estados que têm sistemas de informação com coberturas de grau satisfatório 4 .

Nas últimas décadas, o Ministério da Saúde temrealizadoinvestimentosobjetivandoamelhoria das informações 5,6. Em anos recentes, destacam-se: a regulamentação das atividades desenvolvidas pelas três esferas de governo 7; a divulgação dos dados por meio de CD-ROM e na Internet; a elaboração de publicações técnicas 8,9,10,11; a capacitação de codificadores de causa básica; a contratação de consultores nos estados para o resgate de informações e redução dos óbitos sem definição da causa básica 12; a inclusão de metas relacionadas ao SIM e ao SINASC na Programação Pactuada e Integrada (PPI), colocando o cumprimento das metas como imprescindível à certificação e ao recebimento dos recursos do Teto Financeiro de Epidemiologia e Controle de Doenças. Outros projetos específicos foram também desenvolvidos, como, por exemplo, a estimação da mortalidade infantil para o Brasil 13, o estudo de busca ativa de óbitos infantis em municípios das regiões Norte e Nordeste 14 e a elaboração do Sistema de Monitoramento de Indicadores Relacionados à Mortalidade Infantil (MONITORIMI; http://www.monitorimi.cict. fiocruz.br/metodologia).

As demais instâncias do sistema de saúde, em consonância com a proposição ministerial, têm aderido e agregado experiências locais e regionais 15,16. As experiências dos estados e municípios subsidiaram estratégias de dimensão nacional para a melhoria dos sistemas de informação vitais, tais como a proposição de integração dos dados do SIM e SINASC 10 com o Sistema de Informações da Atenção Básica (SIAB) 17 no âmbito local e projetos para fortalecimento dos sistemas de informação ${ }^{18}$. Além disso, foram incentivados estudos sobre os óbitos infantis em distintas localidades do país, a exemplo do edital específico do Conselho Nacional de Desenvolvimento Científico e Tecnológico (CNPq) financiado pelo Ministério da Saúde.

Ao serem observadas, contudo, as estimativas indiretas do IBGE relativas à cobertura dos óbitos infantis informados ao SIM no Estado de Pernambuco verificam-se coberturas baixas nos últimos anos, inclusive com tendência de decréscimo, sugerindo uma piora na notificação dos dados de mortalidade 10 .

Tendo em vista os investimentos na melhora da qualidade dos dados vitais no Estado de Pernambuco, nos últimos anos, e as tendências em sentido contrário às esperadas, este trabalho tem como objetivo analisar as informações de óbitos e nascimentos quanto à sua adequação, em dois períodos de tempo.

\section{Material e método}

Pernambuco é localizado no centro-leste da Região Nordeste com uma área de $98.937,8 \mathrm{~km}^{2}$, correspondendo a $6,4 \%$ da área do Nordeste brasileiro. Em 2005, a população estimada era de 8.413 .601 de habitantes, representando $16,5 \%$ e $4,7 \%$ da população do Nordeste e do Brasil, respectivamente.

Primeiramente, foram observadas as séries temporais das coberturas estimadas de óbitos de menores de um ano e nascidos vivos no Estado de Pernambuco. Essas coberturas foram obtidas pelo banco de dados dos sistemas de informação disponíveis na página eletrônica do Departamento de Informática do SUS (DATASUS. Indicadores e dados básicos para a saúde, Brasil, 2007. http:// www.datasus.gov.br, acessado em 31/Jan/2009) e pelas estimativas de óbitos e nascidos vivos calculadas pelo IBGE. A proporção entre os dados informados e estimados resultou nas coberturas que foram apresentadas por meio de gráfico. $\mathrm{Na}$ segunda etapa, foram analisados todos os óbitos e nascidos vivos ocorridos no período de 2000 a 2005, informados ao SIM e ao SINASC, respectivamente, de residentes nos 185 municípios do Estado de Pernambuco, incluindo o território de Fernando de Noronha, também disponíveis na página do DATASUS.

O método utilizado para avaliar a adequação das informações sobre mortalidade e nascidos vivos dos municípios foi desenvolvido por Andrade \& Szwarcwald 19. São utilizados os seguintes indicadores: coeficiente de mortalidade geral padronizado por idade, desvio médio relativo do coeficiente geral de mortalidade, razão entre nascidos vivos informados e estimados, desvio médio relativo da taxa de natalidade e proporção de óbitos sem definição da causa básica. Com base nas distribuições desses cinco indicadores nos municípios das Unidades da Federação que têm informações vitais consideradas adequadas (Indicadores e dados básicos para a saúde, Brasil, 2007. http://www.datasus.gov.br, acessado em 
31/Jan/2009), no triênio 2003-2005, foram estabelecidos critérios para classificar os municípios dos demais estados de acordo com a adequação das informações de óbitos e nascimentos, por categoria populacional (menos de 50 mil habitantes; 50 mil habitantes ou mais).

No presente estudo, os pontos de corte para a classificação do município como "satisfatório", "não satisfatório" e "deficiente" em relação à adequação das informações foram definidos pelos percentis $10 \%$ e $1 \%$, para os indicadores coeficiente geral de mortalidade padronizado por idade e razão entre nascidos vivos informados e estimados, e os percentis $90 \%$ e $99 \%$, para os demais indicadores.

Os cinco indicadores foram calculados em dois períodos de tempo, 2000-2002 e 2003-2005, último triênio com informações disponíveis. Para avaliar as coberturas do SIM e do SINASC, foram usados o coeficiente de mortalidade padronizado por idade, utilizando-se a população do Brasil como padrão, e a razão entre nascidos vivos informados e estimados, respectivamente. Os desvios médios relativos serviram para verificar a regularidade das observações nos triênios, enquanto a proporção de óbitos mal definidos foi utilizada como indicador da qualidade das informações de mortalidade.

Todos os municípios de Pernambuco foram classificados como satisfatório, não satisfatório e deficiente, para cada um dos cinco indicadores, nos dois triênios considerados (2000-2002 e 2003-2005), por categoria populacional, utilizando os critérios de adequação estabelecidos. $\mathrm{Na}$ análise, foram considerados dois aspectos: o percentual de municípios e o percentual da população residente. Para fins comparativos, análise similar foi feita para a Região Nordeste e para a totalidade do Brasil.

A visualização da distribuição espacial dos municípios segundo adequação das informações vitais foi realizada mediante uso de mapas temáticos do programa MapInfo (MapInfo Corp., New York, Estados Unidos). Para sintetizar, foram visualizadas três dimensões: causas mal-definidas; mortalidade e natalidade, compostas pela combinação dos indicadores representativos da cobertura e regularidade; além da dimensão de adequação das informações vitais, composta pela combinação dos cinco indicadores.

Para cada uma das três dimensões e da geral, os 185 municípios foram classificados nas seguintes categorias: (i) informações vitais "consolidadas" (quando todos os indicadores da dimensão têm nível satisfatório de adequação); (ii) informações vitais "em fase de consolidação" (quando pelo menos um indicador tem nível não satisfatório, mas nenhum tem nível deficiente); (iii) informações vitais "não consolidadas" (quando pelo menos um indicador tem nível deficiente de adequação).

\section{Resultados}

A Figura 1 evidencia as inconsistências na série temporal das estimativas da cobertura de óbitos em menores de um ano, no Estado de Pernambuco, calculadas como a proporção do número de óbitos notificados ao SIM em relação ao número estimado pelo IBGE. No período de 1991-2004, a maior cobertura estimada correspondeu ao ano de 1993, superior a $80 \%$. Nos anos subseqüentes, a cobertura estimada decresce para um patamar de 65\%, à exceção do ano de 1999, quando alcança $70 \%$. Após flutuações relevantes entre 20002004, o menor valor da série é observado para o ano de 2004 , de $60 \%$.

$\mathrm{Na}$ série temporal da cobertura estimada de nascidos vivos, gerada pela razão entre os nascidos vivos informados ao SINASC e o número de nascidos vivos estimado pelo IBGE, também são encontradas irregularidades, embora não tão intensas quanto as apresentadas pelos dados de mortalidade, cujo maior valor ocorre em 1999, quando atinge $98 \%$, mas decresce para $88 \%$ no ano seguinte (Figura 1).

Na Tabela 1, apresentam-se os critérios de adequação estabelecidos para cada um dos cinco indicadores, por porte populacional do município. Observa-se que, de maneira geral, há mais flexibilidade dos critérios entre os municípios de menor porte. Entre esses, os pontos de corte para os indicadores de cobertura das informações de óbitos e nascimentos são, respectivamente, iguais a 4,39 e 0,64. Entre os municípios com 50 mil habitantes ou mais, os valores limites são de 5,29 e 0,76. Para os indicadores de regularidade, as variações dos limites críticos por categoria populacional são ainda maiores, por conta da maior flutuação dos dados anuais em municípios pequenos. Verifica-se, também, que os critérios de adequação dos desvios médios relativos na taxa de natalidade são mais rigorosos do que os de mortalidade, refletindo a melhor regularidade na informação de nascidos vivos.

Utilizando-se esses critérios, os dados dispostos na Tabela 2 mostram a distribuição dos municípios de Pernambuco, Região Nordeste e Brasil por categoria de adequação das informações de mortalidade. Em geral, o número de municípios e a proporção da população residente em municípios com cobertura, regularidade e proporção de causas mal definidas satisfatórias aumentaram de um triênio para o outro, nos dois grupos de municípios. Em Pernambuco, entre os 


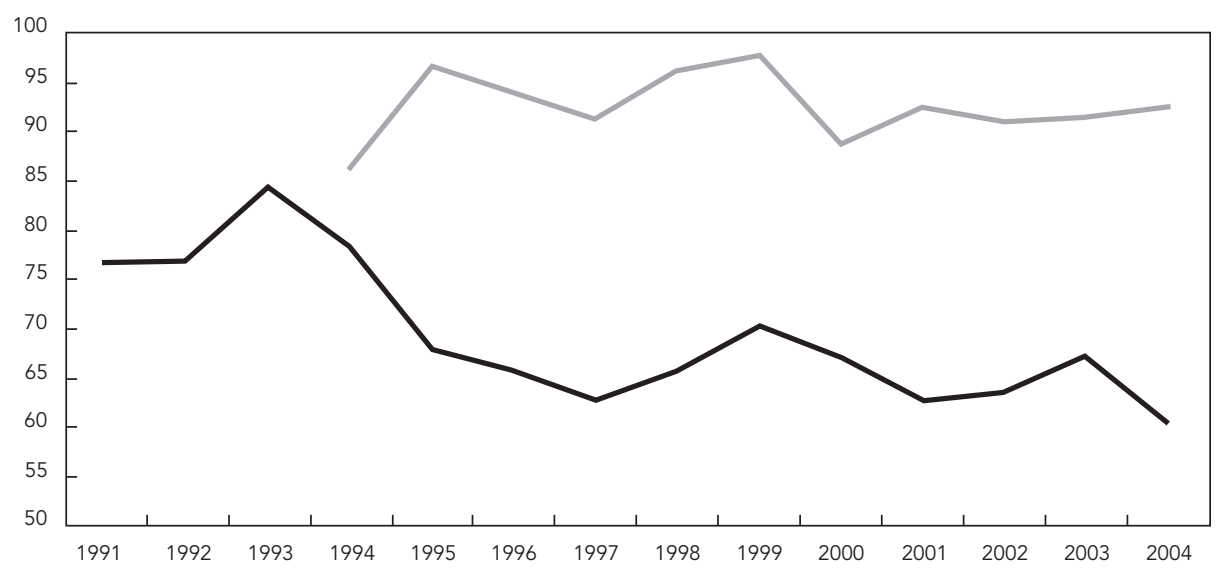

Fonte: Departamento e Informática do SUS (http://www.datasus.gov.br).

Nota: A cobertura estimada do Sistema de Informações sobre Mortalidade (SIM) foi calculada pela proporção de óbitos notificados ao SIM em relação ao número de óbitos estimados pelo Instituto Brasileiro de Geografia e Estatística (IBGE). A cobertura estimada de nascidos vivos foi calculada pela razão entre os nascidos vivos informados ao Sistema de Informações sobre Nascidos Vivos (SINASC) e o número de nascidos vivos estimado pelo IBGE.

municípios com 50 mil habitantes ou mais, no segundo triênio, somente dois municípios não mostraram nível satisfatório de adequação no indicador de cobertura e um no de regularidade.

Ainda considerando-se os municípios com 50 mil habitantes ou mais no segundo triênio, $98 \%$ da população residente estava em municípios com cobertura satisfatória e 99\% em municípios com regularidade satisfatória. Tais percentuais foram maiores que os apresentados para a Região Nordeste, $76 \%$ e $91 \%$, e para o Brasil, $86 \%$ e 93\%, respectivamente. Situação semelhante ocorre nos municípios de menor porte populacional de Pernambuco quando comparados à Região Nordeste e ao Brasil. Comparando as mesmas proporções, para a categoria populacional de menos de 50 mil habitantes, os valores são de $93 \%$ e 99\% em Pernambuco. Para a Região Nordeste, os percentuais são de $54 \%$ e $94 \%$, e para o Brasil, $72 \%$ e $94 \%$, respectivamente, para cobertura e regularidade.

A comparação dos municípios de menor porte populacional com os municípios maiores em Pernambuco evidencia melhores informações entre as cidades com 50 mil habitantes e mais (Tabela 2).

Para os óbitos por causas mal definidas, o padrão encontrado para Pernambuco, Região Nordeste e Brasil, nos dois triênios, nos dois grupos de municípios, foi o de ampliação do número de municípios e da proporção da população residente com informação satisfatória. Apesar dos avanços e de Pernambuco estar em um patamar mais avançado que o Nordeste, o estado ainda tem situação pior que a do Brasil (Tabela 2).

Na Tabela 3, são apresentados os resultados da análise do SINASC para Pernambuco, região Nordeste e Brasil por categoria de adequação dos indicadores de cobertura e regularidade. Ao comparar os dois períodos de tempo, os resultados evidenciam a melhora da regularidade, independentemente do porte populacional, para as três áreas sob estudo. Em relação ao indicador de cobertura, Pernambuco atingiu nível ótimo de adequação, com proporções superiores a 96\%, tanto considerando o número de municípios quanto a proporção da população residente.

As análises feitas para o Nordeste e para o Brasil mostram menores proporções de adequação, quando comparadas às encontradas para o Estado de Pernambuco. Particularmente com relação à cobertura do SINASC, destaca-se a redução, no segundo triênio, da proporção de municípios com 50 mil habitantes ou mais que apresentam cobertura satisfatória (Tabela 3 ).

Na Figura 2, os mapas referentes à adequação do SIM e do SINASC mostram a evolução dos municípios de um triênio para o outro. Com relação ao SIM, os municípios do interior do estado, classificados primeiramente como deficientes, 
Critérios * para classificação dos municípios segundo a adequação das informações vitais por porte populacional.

\begin{tabular}{|c|c|c|c|c|c|c|}
\hline & \multicolumn{3}{|c|}{ Municípios com menos de 50 mil habitantes } & \multicolumn{3}{|c|}{ Municípios com 50 mil habitantes ou mais } \\
\hline & & Critérios & & & Critérios & \\
\hline & Satisfatório & Não satisfatório & Deficiente & Satisfatório & Não satisfatório & Deficiente \\
\hline $\begin{array}{l}\text { Coeficiente geral de mortalidade padroni- } \\
\text { zado por idade }\end{array}$ & $\geq 4,39$ & $\geq 3,42$ e $<4,39$ & $<3,42$ & $\geq 5,29$ & $\geq 4,72$ e $<5,29$ & $<4,72$ \\
\hline $\begin{array}{l}\text { Desvio médio relativo do coeficiente geral } \\
\text { de mortalidade }\end{array}$ & $\leq 21,92$ & $>21,92$ e $\leq 36,37$ & $>36,37$ & $\leq 6,98$ & $>6,98$ e $\leq 9,89$ & $>9,89$ \\
\hline $\begin{array}{l}\text { Razão entre nascidos vivos informados e } \\
\text { estimados }\end{array}$ & $\geq 0,64$ & $\geq 0,47$ e $<0,64$ & $<0,47$ & $\geq 0,76$ & $\geq 0,65$ e $<0,76$ & $<0,65$ \\
\hline Desvio médio relativo da taxa de natalidade & $\leq 16,27$ & $>16,27$ e $\leq 29,58$ & $>29,58$ & $\leq 5,14$ & $>5,14$ e $\leq 8,80$ & $>8,80$ \\
\hline Proporção de óbitos mal definidos & $\leq 17,35$ & $>17,35$ e $\leq 29,37$ & $>29,37$ & $\leq 16,33$ & $>16,33$ e $\leq 22,02$ & $>22,02$ \\
\hline
\end{tabular}

* Critérios obtidos mediante a análise das informações de oito Unidades da Federação (Espírito Santo, Rio de Janeiro, São Paulo, Paraná, Santa Catarina, Rio Grande do Sul, Mato Grosso do Sul e Distrito Federal), consideradas com informações adequadas 31.

evoluíram para não satisfatório, assim como os municípios mais próximos à região metropolitana evoluíram para um nível satisfatório. O SINASC apresenta um quadro ainda melhor, com evolução especialmente nos municípios mais próximos à região metropolitana. O mapa referente à proporção de óbitos por causas mal definidas no último período mostra que a qualidade do preenchimento da causa básica ainda é baixa, tendo progressos apenas em alguns municípios.

Os dois últimos mapas correspondem à distribuição espacial dos municípios com relação à adequação das informações do SIM e do SINASC, em conjunto, para o cálculo direto da mortalidade infantil (Figura 2). Os dados indicam grande melhora, sobretudo naqueles que tinham sistemas não consolidados.

\section{Discussão}

A inconsistência encontrada nas séries temporais das coberturas dos sistemas de informações vitais estimadas pelo IBGE, ao lado do comportamento temporal em sentido contrário aos investimentos para melhorar a qualidade dos dados vitais, é mais uma evidência dos limites das técnicas de mensuração indireta, mesmo em agregados de grande porte populacional, como o Estado de Pernambuco.

Contrariamente, outras formas de aferir a adequação das informações demonstraram avanços inquestionáveis no Estado de Pernambuco, superiores aos alcançados pela Região Nordeste e o Brasil como um todo. Estudos anteriores utilizando os mesmos critérios de avaliação, com pequenas diferenças com relação ao cálculo dos indicadores nos períodos de 1996-1998 12 e 2000200219 enfatizaram a melhoria da qualidade dos dados. O aumento da cobertura do SIM em Pernambuco também foi evidenciado por Paes 20 , utilizando um conjunto de técnicas de mensuração de sub-registro. A melhoria verificada em um período de dez anos (1990-2000) coloca Pernambuco junto aos estados considerados como de boa qualidade da informação 20 .

As tendências díspares obtidas por métodos distintos dificultam as intervenções baseadas em evidências. A evolução temporal da estimativa indireta da cobertura de óbitos infantis, com tendência de decréscimo pode desestimular ações em desenvolvimento e até modificar intervenções que estejam no rumo correto.

Os resultados observados na presente análise indicam que grande parte da população residente em Pernambuco tem informações adequadas tanto de nascimentos quanto de óbitos e que a cobertura de óbitos estimada pelo IBGE em $60 \%$ está provavelmente muito subestimada. Os achados sugerem a necessidade de uma discussão acerca dos métodos adotados pelo Ministério da Saúde para o cálculo da mortalidade infantil e da cobertura das informações vitais.

Quanto ao método utilizado no presente trabalho para avaliar a adequação das informações, é necessário destacar algumas limitações. Em primeiro lugar, no que se refere à cobertura, os indicadores adotados são mais excludentes do que qualificadores das informações, pois eles servem bem para identificar os municípios com deficiências, mas são insuficientes para estabelecer as coberturas dos sistemas. A definição de 
Tabela 2

Percentual de municípios e de população residente por categoria de adequação das informações de mortalidade (Sistema de Informações sobre Mortalidade - SIM) de acordo com o porte populacional e período de tempo. Pernambuco, Região Nordeste e Brasil, $2000-2002$ e $2003-2005$.

\begin{tabular}{|c|c|c|c|c|c|c|c|c|c|c|c|c|c|}
\hline \multirow[t]{4}{*}{ Indicadores } & & \multicolumn{12}{|c|}{ Pernambuco } \\
\hline & & \multicolumn{6}{|c|}{ Municípios com menos de 50 mil habitantes } & \multicolumn{6}{|c|}{ Municípios com 50 mil habitantes ou mais } \\
\hline & & \multicolumn{3}{|c|}{$2000-2002$} & \multicolumn{3}{|c|}{$2003-2005$} & \multicolumn{3}{|c|}{$2000-2002$} & \multicolumn{3}{|c|}{ 2003-2005 } \\
\hline & & $\begin{array}{l}\text { Satis- } \\
\text { fatório }\end{array}$ & $\begin{array}{c}\text { Não } \\
\text { satis- } \\
\text { fatório }\end{array}$ & $\begin{array}{l}\text { Defi- } \\
\text { ciente }\end{array}$ & $\begin{array}{l}\text { Satis- } \\
\text { fatório }\end{array}$ & $\begin{array}{c}\text { Não } \\
\text { satis- } \\
\text { fatório }\end{array}$ & $\begin{array}{l}\text { Defi- } \\
\text { ciente }\end{array}$ & $\begin{array}{l}\text { Satis- } \\
\text { fatório }\end{array}$ & $\begin{array}{c}\text { Não } \\
\text { satis- } \\
\text { fatório }\end{array}$ & $\begin{array}{l}\text { Defi- } \\
\text { ciente }\end{array}$ & $\begin{array}{l}\text { Satis- } \\
\text { fatório }\end{array}$ & $\begin{array}{c}\text { Não } \\
\text { satis- } \\
\text { fatório }\end{array}$ & $\begin{array}{l}\text { Defi- } \\
\text { ciente }\end{array}$ \\
\hline Cobertura & Municípios (n) & 131 & 16 & 6 & 137 & 13 & 3 & 30 & 1 & 1 & 30 & 2 & - \\
\hline \multirow[t]{2}{*}{ SIM } & Municípios (\%) & 85,6 & 10,5 & 3,9 & 89,5 & 8,5 & 2,0 & 93,8 & 3,1 & 3,1 & 93,8 & 6,3 & - \\
\hline & População (\%) & 88,6 & 9,1 & 2,3 & 92,9 & 5,8 & 1,3 & 97,5 & 1,4 & 1,1 & 97,9 & 2,1 & - \\
\hline Regularidade & Municípios (n) & 140 & 13 & - & 148 & 5 & - & 25 & 5 & 2 & 31 & 1 & - \\
\hline \multirow[t]{2}{*}{ SIM } & Municípios (\%) & 91,5 & 8,5 & - & 96,7 & 3,3 & - & 78,1 & 15,6 & 6,3 & 96,9 & 3,1 & - \\
\hline & População (\%) & 95,3 & 4,7 & - & 98,9 & 1,1 & - & 91,5 & 6,3 & 2,2 & 99,0 & 1,0 & - \\
\hline Óbitos mal & Municípios (n) & 13 & 25 & 115 & 47 & 37 & 69 & 14 & 2 & 16 & 20 & 3 & 9 \\
\hline \multirow[t]{2}{*}{ definidos } & Municípios (\%) & 8,5 & 16,3 & 75,2 & 30,7 & 24,2 & 45,1 & 43,8 & 6,3 & 50,0 & 62,5 & 9,4 & 28,1 \\
\hline & População (\%) & 7,9 & 16,4 & 75,7 & 27,4 & 27,5 & 45,1 & 69,9 & 2,1 & 28,0 & 80,8 & 7,2 & 12,0 \\
\hline
\end{tabular}

Região Nordeste

\begin{tabular}{|c|c|c|c|c|c|c|c|c|c|c|c|c|c|}
\hline \multirow[t]{3}{*}{ Indicadores } & & \multicolumn{6}{|c|}{ Municípios com menos de 50 mil habitantes } & \multicolumn{6}{|c|}{ Municípios com 50 mil habitantes ou mais } \\
\hline & & \multicolumn{3}{|c|}{$2000-2002$} & \multicolumn{3}{|c|}{ 2003-2005 } & \multicolumn{3}{|c|}{ 2000-2002 } & \multicolumn{3}{|c|}{ 2003-2005 } \\
\hline & & $\begin{array}{l}\text { Satis- } \\
\text { fatório }\end{array}$ & $\begin{array}{c}\text { Não } \\
\text { satis- } \\
\text { fatório }\end{array}$ & $\begin{array}{l}\text { Defi- } \\
\text { ciente }\end{array}$ & $\begin{array}{l}\text { Satis- } \\
\text { fatório }\end{array}$ & $\begin{array}{c}\text { Não } \\
\text { satis- } \\
\text { fatório }\end{array}$ & $\begin{array}{l}\text { Defi- } \\
\text { ciente }\end{array}$ & $\begin{array}{l}\text { Satis- } \\
\text { fatório }\end{array}$ & $\begin{array}{c}\text { Não } \\
\text { satis- } \\
\text { fatório }\end{array}$ & $\begin{array}{l}\text { Defi- } \\
\text { ciente }\end{array}$ & $\begin{array}{l}\text { Satis- } \\
\text { fatório }\end{array}$ & $\begin{array}{c}\text { Não } \\
\text { satis- } \\
\text { fatório }\end{array}$ & $\begin{array}{l}\text { Defi- } \\
\text { ciente }\end{array}$ \\
\hline Cobertura & Municípios (n) & 656 & 507 & 480 & 857 & 540 & 245 & 73 & 27 & 44 & 85 & 30 & 35 \\
\hline \multirow[t]{2}{*}{ SIM } & Municípios (\%) & 39,9 & 30,9 & 29,2 & 52,2 & 32,9 & 14,9 & 50,7 & 18,8 & 30,6 & 56,7 & 20,0 & 23,3 \\
\hline & População (\%) & 45,0 & 28,9 & 26,1 & 54,1 & 31,7 & 14,2 & 71,3 & 14,6 & 14,1 & 75,7 & 14,5 & 9,8 \\
\hline Regularidade & Municípios (n) & 1.255 & 256 & 132 & 1.452 & 165 & 25 & 97 & 19 & 28 & 122 & 17 & 11 \\
\hline \multirow[t]{2}{*}{ SIM } & Municípios (\%) & 76,4 & 15,6 & 8,0 & 88,4 & 10,0 & 1,5 & 67,4 & 13,2 & 19,4 & 81,3 & 11,3 & 7,3 \\
\hline & População (\%) & 83,6 & 11,0 & 5,5 & 93,9 & 5,2 & 0,9 & 84,8 & 5,9 & 9,3 & 91,3 & 5,2 & 3,5 \\
\hline Óbitos mal & Municípios (n) & 177 & 258 & 1208 & 394 & 355 & 893 & 37 & 19 & 88 & 57 & 21 & 72 \\
\hline \multirow[t]{2}{*}{ definidos } & Municípios (\%) & 10,8 & 15,7 & 73,5 & 24,0 & 21,6 & 54,4 & 25,7 & 13,2 & 61,1 & 38,0 & 14,0 & 48,0 \\
\hline & População (\%) & 11,8 & 17,6 & 70,6 & 22,7 & 23,3 & 54,0 & 45,3 & 21,1 & 33,6 & 56,9 & 9,4 & 33,6 \\
\hline
\end{tabular}

Indicadores
Municípios com menos de 50 mil habitantes

2000-2002

2003-2005

Não

ciente fatório satis- ciente fatório

fatório

fatório

\begin{tabular}{|c|c|c|c|c|c|c|c|c|c|c|c|c|c|}
\hline & & $\begin{array}{l}\text { Satis- } \\
\text { fatório }\end{array}$ & $\begin{array}{c}\text { Não } \\
\text { satis- } \\
\text { fatório }\end{array}$ & $\begin{array}{l}\text { Defi- } \\
\text { ciente }\end{array}$ & $\begin{array}{l}\text { Satis- } \\
\text { fatório }\end{array}$ & $\begin{array}{c}\text { Não } \\
\text { satis- } \\
\text { fatório }\end{array}$ & $\begin{array}{l}\text { Defi- } \\
\text { ciente }\end{array}$ & $\begin{array}{l}\text { Satis- } \\
\text { fatório }\end{array}$ & $\begin{array}{c}\text { Não } \\
\text { satis- } \\
\text { fatório }\end{array}$ & $\begin{array}{l}\text { Defi- } \\
\text { ciente }\end{array}$ & $\begin{array}{l}\text { Satis- } \\
\text { fatório }\end{array}$ & $\begin{array}{c}\text { Não } \\
\text { satis- } \\
\text { fatório }\end{array}$ & $\begin{array}{l}\text { Defi- } \\
\text { ciente }\end{array}$ \\
\hline Cobertura & Municípios (n) & 2.993 & 1.090 & 892 & 3.475 & 1.032 & 496 & 393 & 69 & 70 & 419 & 77 & 61 \\
\hline \multirow[t]{2}{*}{ SIM } & Municípios (\%) & 60,2 & 21,9 & 17,9 & 69,5 & 20,6 & 9,9 & 73,9 & 13,0 & 13,2 & 75,2 & 13,8 & 11,0 \\
\hline & População (\%) & 65,3 & 18,4 & 16,3 & 72,4 & 18,1 & 9,5 & 84,5 & 10,3 & 5,2 & 85,7 & 10,1 & 4,2 \\
\hline Regularidade & Municípios (n) & 3.989 & 694 & 292 & 4.321 & 570 & 112 & 443 & 38 & 51 & 467 & 64 & 26 \\
\hline \multirow[t]{2}{*}{ SIM } & Municípios (\%) & 80,2 & 13,9 & 5,9 & 86,4 & 11,4 & 2,2 & 83,3 & 7,1 & 9,6 & 83,8 & 11,5 & 4,7 \\
\hline & População (\%) & 88,0 & 8,4 & 3,6 & 94,2 & 5,0 & 0,8 & 93,8 & 2,6 & 3,7 & 93,0 & 4,9 & 2,1 \\
\hline \multirow{3}{*}{$\begin{array}{l}\text { Óbitos mal } \\
\text { definidos }\end{array}$} & Municípios (n) & 2.454 & 815 & 1.706 & 3.034 & 767 & 1.202 & 352 & 58 & 122 & 394 & 60 & 103 \\
\hline & Municípios (\%) & 49,3 & 16,4 & 34,3 & 60,6 & 15,3 & 24,0 & 66,2 & 10,9 & 22,9 & 70,7 & 10,8 & 18,5 \\
\hline & População (\%) & 47,1 & 16,3 & 36,6 & 55,7 & 16,7 & 27,6 & 78,9 & 11,0 & 10,0 & 82,5 & 8,0 & 9,6 \\
\hline
\end{tabular}

Municípios com 50 mil habitantes ou mais

2000-2002

2003-2005 
Percentual de municípios e de população residente por categoria de adequação das informações de nascidos vivos (Sistema de Informações sobre Nascidos Vivos - SINASC) de acordo com o porte populacional e período de tempo. Pernambuco, Região Nordeste e Brasil, $2000-2002$ e $2003-2005$.

\begin{tabular}{|c|c|c|c|c|c|c|c|c|c|c|c|c|c|}
\hline \multirow[t]{4}{*}{ Indicadores } & & \multicolumn{12}{|c|}{ Pernambuco } \\
\hline & & \multicolumn{6}{|c|}{ Municípios com menos de 50 mil habitantes } & \multicolumn{6}{|c|}{ Municípios com 50 mil habitantes ou mais } \\
\hline & & \multicolumn{3}{|c|}{$2000-2002$} & \multicolumn{3}{|c|}{ 2003-2005 } & \multicolumn{3}{|c|}{$2000-2002$} & \multicolumn{3}{|c|}{ 2003-2005 } \\
\hline & & $\begin{array}{l}\text { Satis- } \\
\text { fatório }\end{array}$ & $\begin{array}{c}\text { Não } \\
\text { satis- } \\
\text { fatório }\end{array}$ & $\begin{array}{l}\text { Defi- } \\
\text { ciente }\end{array}$ & $\begin{array}{l}\text { Satis- } \\
\text { fatório }\end{array}$ & $\begin{array}{c}\text { Não } \\
\text { satis- } \\
\text { fatório }\end{array}$ & $\begin{array}{l}\text { Defi- } \\
\text { ciente }\end{array}$ & $\begin{array}{l}\text { Satis- } \\
\text { fatório }\end{array}$ & $\begin{array}{c}\text { Não } \\
\text { satis- } \\
\text { fatório }\end{array}$ & $\begin{array}{l}\text { Defi- } \\
\text { ciente }\end{array}$ & $\begin{array}{l}\text { Satis- } \\
\text { fatório }\end{array}$ & $\begin{array}{c}\text { Não } \\
\text { satis- } \\
\text { fatório }\end{array}$ & $\begin{array}{l}\text { Defi- } \\
\text { ciente }\end{array}$ \\
\hline Cobertura & Municípios (n) & 150 & 1 & 2 & 147 & 4 & 2 & 31 & 1 & - & 31 & 1 & - \\
\hline \multirow[t]{2}{*}{ SINASC } & Municípios (\%) & 98,0 & 0,7 & 1,3 & 96,1 & 2,6 & 1,3 & 96,9 & 3,1 & - & 96,9 & 3,1 & - \\
\hline & População (\%) & 98,7 & 1,0 & 0,3 & 96,9 & 2,0 & 1,1 & 99,0 & 1,0 & - & 98,7 & 1,3 & - \\
\hline Regularidade & Municípios (n) & 145 & 6 & 2 & 150 & 3 & - & 18 & 11 & 3 & 27 & 5 & - \\
\hline \multirow[t]{3}{*}{ SINASC } & Municípios (\%) & 94,8 & 3,9 & 1,3 & 98,0 & 2,0 & - & 56,3 & 34,4 & 9,4 & 84,4 & 15,6 & - \\
\hline & População (\%) & 96,4 & 3,3 & 0,3 & 98,6 & 1,4 & - & 61,5 & 35,1 & 3,4 & 93,7 & 6,3 & - \\
\hline & & \multicolumn{12}{|c|}{ Região Nordeste } \\
\hline \multirow[t]{3}{*}{ Indicadores } & & \multicolumn{6}{|c|}{ Municípios com menos de 50 mil habitantes } & \multicolumn{6}{|c|}{ Municípios com 50 mil habitantes ou mais } \\
\hline & & \multicolumn{3}{|c|}{$2000-2002$} & \multicolumn{3}{|c|}{ 2003-2005 } & \multicolumn{3}{|c|}{$2000-2002$} & \multicolumn{3}{|c|}{ 2003-2005 } \\
\hline & & $\begin{array}{l}\text { Satis- } \\
\text { fatório }\end{array}$ & $\begin{array}{c}\text { Não } \\
\text { satis- } \\
\text { fatório }\end{array}$ & $\begin{array}{l}\text { Defi- } \\
\text { ciente }\end{array}$ & $\begin{array}{l}\text { Satis- } \\
\text { fatório }\end{array}$ & $\begin{array}{c}\text { Não } \\
\text { satis- } \\
\text { fatório }\end{array}$ & $\begin{array}{l}\text { Defi- } \\
\text { ciente }\end{array}$ & $\begin{array}{l}\text { Satis- } \\
\text { fatório }\end{array}$ & $\begin{array}{c}\text { Não } \\
\text { satis- } \\
\text { fatório }\end{array}$ & $\begin{array}{l}\text { Defi- } \\
\text { ciente }\end{array}$ & $\begin{array}{l}\text { Satis- } \\
\text { fatório }\end{array}$ & $\begin{array}{c}\text { Não } \\
\text { satis- } \\
\text { fatório }\end{array}$ & $\begin{array}{l}\text { Defi- } \\
\text { ciente }\end{array}$ \\
\hline Cobertura & Municípios (n) & 1.402 & 174 & 67 & 1.472 & 143 & 27 & 131 & 3 & 10 & 128 & 17 & 5 \\
\hline \multirow[t]{2}{*}{ SINASC } & Municípios (\%) & 85,3 & 10,6 & 4,1 & 89,6 & 8,7 & 1,6 & 91,0 & 2,1 & 6,9 & 85,3 & 11,3 & 3,3 \\
\hline & População (\%) & 87,3 & 9,3 & 3,4 & 90,8 & 7,9 & 1,3 & 95,8 & 0,8 & 3,3 & 91,3 & 6,3 & 2,4 \\
\hline Regularidade & Municípios (n) & 1.338 & 219 & 86 & 1.551 & 88 & 3 & 70 & 44 & 30 & 125 & 21 & 4 \\
\hline \multirow[t]{3}{*}{ SINASC } & Municípios (\%) & 81,4 & 13,3 & 5,2 & 94,5 & 5,4 & 0,2 & 48,6 & 30,6 & 20,8 & 83,3 & 14,0 & 2,7 \\
\hline & População (\%) & 86,0 & 9,9 & 4,1 & 96,7 & 3,2 & 0,1 & 58,1 & 31,3 & 10,6 & 92,0 & 7,1 & 0,9 \\
\hline & & \multicolumn{12}{|c|}{ Brasil } \\
\hline \multirow[t]{3}{*}{ Indicadores } & & \multicolumn{6}{|c|}{ Municípios com menos de 50 mil habitantes } & \multicolumn{6}{|c|}{ Municípios com 50 mil habitantes ou mais } \\
\hline & & \multicolumn{3}{|c|}{$2000-2002$} & \multicolumn{3}{|c|}{ 2003-2005 } & \multicolumn{3}{|c|}{$2000-2002$} & & $2003-200$ & \\
\hline & & $\begin{array}{l}\text { Satis- } \\
\text { fatório }\end{array}$ & $\begin{array}{c}\text { Não } \\
\text { satis- } \\
\text { fatório }\end{array}$ & $\begin{array}{l}\text { Defi- } \\
\text { ciente }\end{array}$ & $\begin{array}{l}\text { Satis- } \\
\text { fatório }\end{array}$ & $\begin{array}{c}\text { Não } \\
\text { satis- } \\
\text { fatório }\end{array}$ & $\begin{array}{l}\text { Defi- } \\
\text { ciente }\end{array}$ & $\begin{array}{c}\text { Satis- } \\
\text { fatório }\end{array}$ & $\begin{array}{c}\text { Não } \\
\text { satis- } \\
\text { fatório }\end{array}$ & $\begin{array}{l}\text { Defi- } \\
\text { ciente }\end{array}$ & $\begin{array}{l}\text { Satis- } \\
\text { fatório }\end{array}$ & $\begin{array}{c}\text { Não } \\
\text { satis- } \\
\text { fatório }\end{array}$ & $\begin{array}{l}\text { Defi- } \\
\text { ciente }\end{array}$ \\
\hline Cobertura & Municípios (n) & 4.374 & 447 & 154 & 4.365 & 543 & 95 & 507 & 7 & 18 & 483 & 58 & 16 \\
\hline SINASC & Municípios (\%) & 87,9 & 9,0 & 3,1 & 87,2 & 10,9 & 1,9 & 95,3 & 1,3 & 3,4 & 86,7 & 10,4 & 2,9 \\
\hline & População (\%) & 89,8 & 7,4 & 2,8 & 90,7 & 8,0 & 1,4 & 98,3 & 0,4 & 1,2 & 93,0 & 5,7 & 1,3 \\
\hline Regularidade & Municípios (n) & 4.108 & 656 & 211 & 4.569 & 391 & 43 & 256 & 210 & 66 & 467 & 78 & 12 \\
\hline SINASC & Municípios (\%) & 82,6 & 13,2 & 4,2 & 91,3 & 7,8 & 0,9 & 48,1 & 39,5 & 12,4 & 83,8 & 14,0 & 2,2 \\
\hline & População (\%) & 89,0 & 8,0 & 3,1 & 96,3 & 3,5 & 0,3 & 59,1 & 35,8 & 5,1 & 91,6 & 7,5 & 0,9 \\
\hline
\end{tabular}

critérios únicos nacionais, ao invés de regionais, é outra limitação do método, tendo em vista a diversidade dos padrões demográficos existentes no país. Além disso, como os indicadores dependem de projeções populacionais, os resultados são afetados pelas estimativas populacionais por grupo de idade, que ainda são baseadas no último censo.

No caso de menores de um ano de idade, a projeção populacional é ainda mais afetada, já que a taxa de fecundidade tem tido ritmo acelerado de decréscimo em anos recentes. A queda 
Figura 2

Classificação do município quanto à adequação das informações de mortalidade, nascidos vivos e proporções de óbitos por causas mal definidas. Pernambuco, Brasil, 2000-2005.

2a) Óbitos (2000-2002)

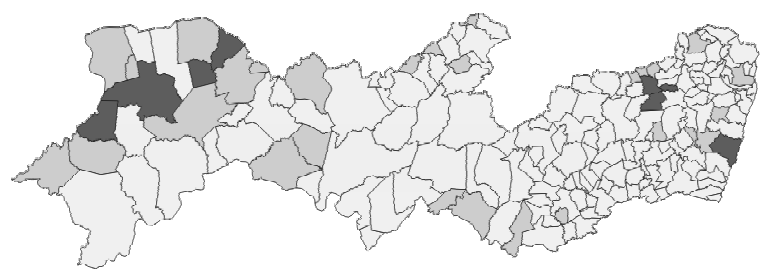

2c) Nascidos vivos (2000-2002)

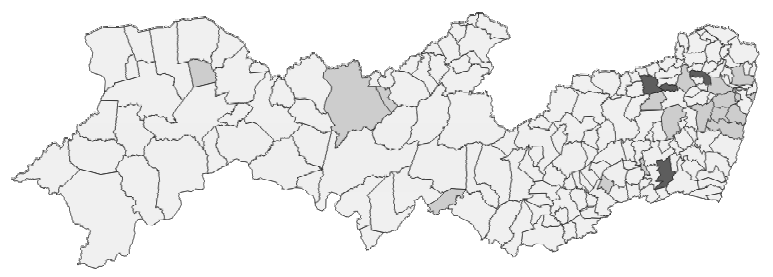

2e) Óbitos por causas mal definidas (2000-2002)

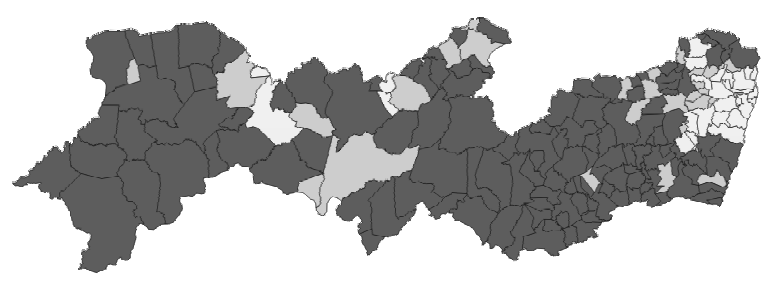

2g) Óbitos e nascidos vivos (2000-2002)

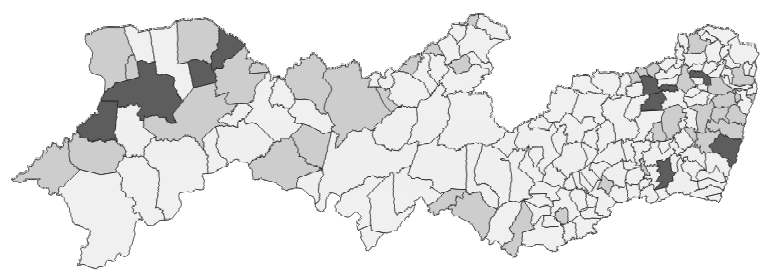

2b) Óbitos (2003-2005)

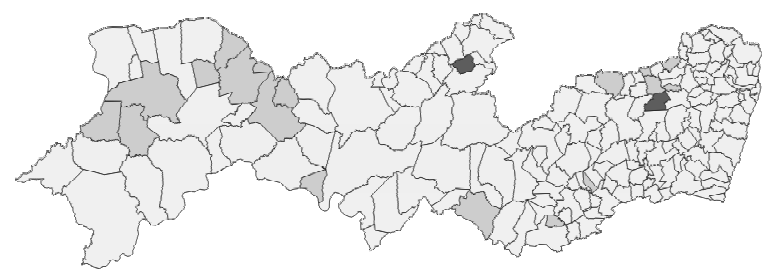

2d) Nascidos vivos (2003-2005)

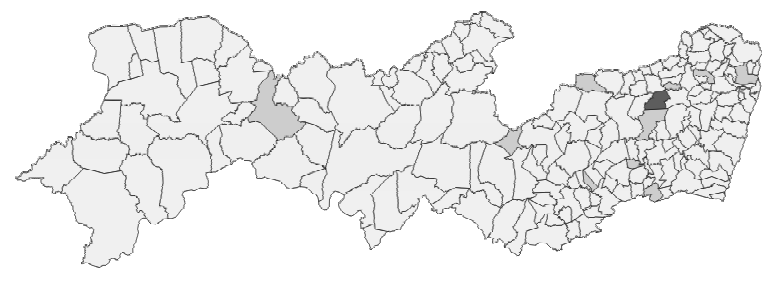

2f) Óbitos por causas mal definidas (2003-2005)

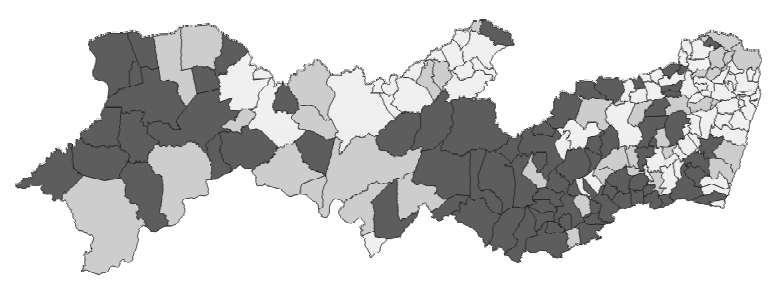

2h) Óbitos e nascidos vivos (2003-2005)

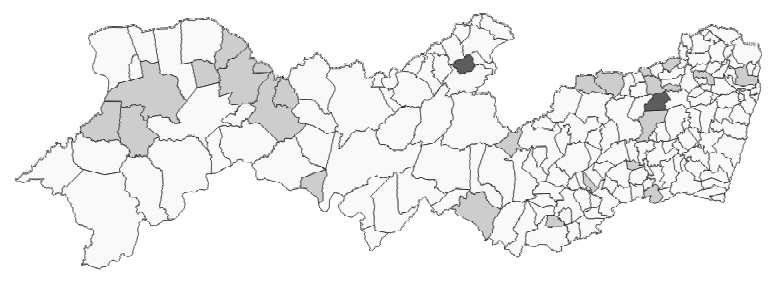

Satisfatório

Não satisfatório

Deficiente 
na fecundidade também é um fator a ser considerado quando analisada a aparente tendência de queda observada para a cobertura do SINASC em alguns grupos de municípios. Esta queda pode ser conseqüência de uma sobrestimação dos nascidos vivos, resultando em valores mais baixos da razão entre nascidos vivos informados e estimados no segundo triênio.

Apesar de fazer parte do SIM, na abordagem aqui utilizada, o percentual de causas mal definidas é avaliado, separadamente, por causa de sua importância como parâmetro para avaliação da qualidade das informações de mortalidade. Como foi constatada no estudo, a adequação dessa dimensão ainda se encontra em patamares bastante inferiores aos alcançados pela cobertura e regularidade do sistema.

O elevado percentual de causas mal definidas restringe o conhecimento do perfil nosológico de mortalidade e a comparação das taxas de mortalidade infantil por causa em diferentes localidades. A utilização da distribuição proporcional por causa entre os óbitos bem definidos, estratégia utilizada para solucionar o problema, foi avaliada como insuficiente para a construção do perfil da mortalidade 21 . A inadequação desse procedimento já havia sido verificada em estudo realizado por Mello-Jorge et al. ${ }^{22}$, dadas as diferenças encontradas após a investigação de um conjunto de óbitos classificados como mal definidos.

Os resultados apresentados evidenciam a melhoria no preenchimento da causa básica de morte, apesar de Pernambuco e Região Nordeste ainda manterem elevados percentuais de óbitos por causas mal definidas. É importante destacar que as altas proporções desse indicador vão além dos investimentos na qualidade do sistema de informação. A definição da causa básica de óbito tem forte relação com o acesso à assistência médica no momento da morte. Nos municípios com 50 mil habitantes ou mais, onde se concentra grande parte dos serviços de saúde, a proporção de mal definidos é menor.

Evidências fortalecendo a relação entre a proporção de óbitos por causas mal definidas e a desigualdade no acesso aos serviços de saúde são apresentadas em estudos que mostram elevado percentual de causas mal definidas entre óbitos domiciliares e óbitos hospitalares ocorridos em emergências de grandes hospitais 23. Elevados percentuais são observados de forma geral em regiões rurais de países em desenvolvimento 23,24,25,26

Desconsiderar estas questões e pretender resolver as dificuldades de definição da causa básica relacionando-as apenas aos sistemas de informação, no cenário atual de algumas regiões brasileiras, é negar as iniqüidades na prestação de serviços nas várias dimensões de acesso consideradas 27 . A disponibilidade de serviços no momento e lugar da necessidade do usuário tem sido relatada como uma das expressões da desigualdade na oferta de serviços, na atenção primária e secundária à saúde no país 28,29 . Por outro lado, a possibilidade de obtenção do cuidado de acordo com a capacidade de compra do usuário, seja pela dificuldade de transporte, ou as atitudes de provedores e prestadores de serviços têm dificultado o acesso de grupos mais vulneráveis à assistência.

Ao mesmo tempo, outras evidências alertam para outros fatores a serem considerados. Estudos também têm identificado problemas no preenchimento de Declarações de Óbito (DO) hospitalares, pela omissão de informações disponíveis nos prontuários bem como inadequação no fluxo de DO, impedindo a incorporação de informações presentes nos laudos das necropsias $14,22,23,30$.

As rápidas mudanças demográficas e de saúde ocorridas e a necessidade de diferenciar estágios de implementação dos sistemas de informações levam ao desenvolvimento de métodos e técnicas que possibilitem avaliar os sistemas de informações, com fins de propor intervenções de acordo com as fragilidades loco-regionais.

Apesar das limitações do método aqui exposto, os aspectos levantados neste estudo ressaltam a melhora dos sistemas de informação ao mesmo tempo em que destacam os desafios a serem enfrentados. A análise realizada no Estado de Pernambuco aponta para a importância da análise das informações sobre os eventos vitais no nível do município, permitindo avaliar os investimentos feitos para a melhoria das informações e a tendência temporal da mortalidade infantil de forma mais realística. 


\section{Resumo}

O Ministério da Saúde tem realizado importante investimento na melhoria da qualidade dos sistemas de informação sobre mortalidade e nascidos vivos. Entretanto, na maioria dos estados brasileiros a mortalidade infantil ainda é calculada por métodos indiretos, sem levar em consideração os avanços ocorridos nessa área. O objetivo deste artigo é avaliar a adequação das informações de óbitos e nascimentos nos municípios de Pernambuco, Brasil, utilizando indicadores de cobertura e regularidade dos sistemas. Os resultados evidenciaram as inconsistências na série temporal das estimativas por métodos indiretos da cobertura de óbitos infantis no estado. Os indicadores de adequação apontaram uma importante melhora da cobertura e regularidade das informações vitais. A proporção de óbitos por causas mal definidas, indicador da qualidade dos dados, mostrou também melhoras, embora em grau menor. A evolução observada em Pernambuco aponta para a importância de uma reflexão acerca da utilização de métodos diretos para o cálculo da mortalidade infantil, pelo menos nos municípios com informações adequadas.

Sistemas de Informação; Nascimento Vivo; Mortalidade Infantil; Avaliação em Saúde

\section{Colaboradores}

P. G. Frias participou da concepção, pesquisa bibliográfica, análise dos dados e redação do artigo. P. M. H. Pereira colaborou na concepção, pesquisa bibliográfica e redação do artigo. C. L. T. Andrade contribuiu na concepção, construção do banco, análise dos dados e revisão do artigo. P. I. C. Lira participou da revisão final do texto. C. L. Szwarcwald colaborou na concepção, análise dos dados, redação e revisão final do artigo.

\section{Referências}

1. Mello-Jorge MH, Laurenti R, Gotlieb SLD. Análise da qualidade das estatísticas vitais brasileiras: a experiência de implantação do SIM e do SINASC. Ciênc Saúde Coletiva 2007; 12:643-54.

2. Rede Interagencial de Informações para a Saúde. Indicadores básicos para saúde no Brasil: conceitos e aplicações. 2a Ed. Brasília: Organização PanAmericana da Saúde; 2008.

3. Murray CJ, Laakso T, Shibuya K, Hill K, Lopez AD. Can we achieve Millennium Development Goal 4 ? New analysis of country trends and forecasts of under-5 mortality to 2015 . Lancet $2007 ; 370$ : 1040-54.
4. Szwarcwald CL, Leal MC, Andrade CLT, Souza Jr. PRB. Estimação da mortalidade infantil no Brasil: o que dizem as informações sobre óbitos e nascimentos do Ministério da Saúde? Cad Saúde Pública 2002; 18:1725-36.

5. Ministério da Saúde. Sistemas de informação - SIM e SINASC. In: Anais 2a Expoepi - Mostra Nacional de Experiências Bem-sucedidas em Epidemiologia, Prevenção e Controle de Doenças. Relatório das Oficinas de Trabalho. Brasília: Ministério da Saúde; 2003. p. 31-6. (Série D. Reuniões e Conferências). 
6. Ministério da Saúde. Monitoramento da acurácia dos sistemas de informações sobre mortalidade e nascidos vivos. In: Anais 3a Expoepi Mostra Nacional de Experiências Bem-sucedidas em Epidemiologia, Prevenção e Controle de Doenças. Brasília: Ministério da Saúde, 2004. p. 173-80. (Série D. Reuniões e Conferências).

7. Ministério da Saúde. Portaria $n^{\circ} .20$, de 3 de outubro de 2003. Regulamenta a coleta de dados, fluxo e periodicidade de envio das informações sobre óbitos e nascidos vivos para os Sistemas de Informação sobre Mortalidade - SIM - e sobre Nascidos Vivos - SINASC. Diário Oficial da União 2003; 7 out.

8. Fundação Nacional de Saúde, Ministério da Saúde. Manual de instruções para o preenchimento da Declaração de Nascido Vivo. 3a Ed. Brasília: Fundação Nacional de Saúde, Ministério da Saúde; 2001.

9. Fundação Nacional de Saúde, Ministério da Saúde. Manual de instruções para o preenchimento da Declaração de Óbito. 3ạ Ed. Brasília: Fundação Nacional de Saúde, Ministério da Saúde; 2001.

10. Ministério da Saúde. Sistemas de Informações sobre Mortalidade (SIM) e Nascidos Vivos (SINASC) para os profissionais do Programa Saúde da Família. 2a Ed. Rev. Brasília: Ministério da Saúde; 2004. (Série F. Comunicação e Educação em Saúde).

11. Conselho Federal de Medicina, Ministério da Saúde. A Declaração de Óbito: documento necessário e importante. Brasília: Ministério da Saúde; 2006 (Série A. Normas e Manuais Técnicos).

12. Ministério da Saúde. Manual para investigação do óbito com causa mal definida. Brasília: Ministério da Saúde; 2008. (Série A. Normas e Manuais Técni$\cos )$.

13. Centro de Informação Científica e Tecnológica, Fundação Oswaldo Cruz. Projeto para estimação da mortalidade infantil no Brasil no ano de 2000: relatório final. Rio de Janeiro: Centro de Informação Científica e Tecnológica, Fundação Oswaldo Cruz; 2002.

14. Frias PG, Pereira PMH, Andrade CLT, Szwarcwald CL. Sistema de Informações sobre Mortalidade: estudo de caso em municípios com precariedade dos dados. Cad Saúde Pública 2008; 24:2257-66.

15. Secretaria de Saúde de Pernambuco. Coletânea de projetos relacionados com a redução da mortalidade infantil. Recife: Secretaria de Saúde de Pernambuco; 1997. (Cadernos de Avaliação. Série Projeto Salva-Vidas, 7).

16. Ministério da Saúde. SIM e SINASC - web \& agente de saúde eletrônico: uma parceria que faz a diferença promovendo mais agilidade, flexibilidade e segurança à vigilância epidemiológica de Fortaleza - CE. In: Anais 8a Expoepi - Mostra Nacional de Experiências Bem-sucedidas em Epidemiologia, Prevenção e Controle de Doenças. Brasília: Ministério da Saúde, 2008. p. 23-4. (Série D. Reuniões e Conferências).

17. Mello-Jorge MHP, Gotlieb SLD. O Sistema de Informação de Atenção Básica como fonte de dados para os sistemas de informações sobre mortalidade e sobre nascidos vivos. Inf Epidemiol SUS 2000; 10:7-18.
18. Secretaria de Vigilância em Saúde, Ministério da Saúde. Projeto Vigisus II. Subcomponente IV. Fortalecimento institucional da capacidade de gestão em vigilância em saúde nos estados e municípios. 2004. http://portal.saude.gov.br/portal/arquivos/ pdf/vigisusII.pdf (acessado em 31/Dez/2008).

19. Andrade CLT, Szwarcwald CL. Desigualdades sócio-espaciais da adequação das informações de nascimentos e óbitos do Ministério da Saúde, Brasil, 2000-2002. Cad Saúde Pública 2007; 23: 1207-16.

20. Paes N. Avaliação da cobertura dos registros de óbitos dos estados brasileiros em 2000. Rev Saúde Pública 2005; 39:882-90.

21. Cavalini LT, Ponce de Leon ACM. Correção de subregistro de óbitos e proporção de internações por causas mal definidas. Rev Saúde Pública 2007; 41:85-93.

22. Mello-Jorge MHP, Gotlieb SLD, Laurenti R. O sistema de informações sobre mortalidade: problemas e propostas para seu enfrentamento. II - Mortes por causas externas. Rev Bras Epidemiolol 2002; 5:212-23.

23. Haraki CAP, Gotlieb SLD, Laurenti R. Confiabilidade do Sistema de Informações sobre Mortalidade em município do sul do Estado de São Paulo. Rev Bras Epidemiol 2005; 8:19-24.

24. Mathers CD, Fat DM, Inoue M, Rao C, Lopez AD. Counting the dead and what they did from: an assessment of the global status of cause of death data. Bull World Health Organ 2005; 83:171-7.

25. Setel PW, Sankoh O, Rao C, Velkoff VA, Mathers C, Gonghuan Y, et al. Sample registration of vital events with verbal autopsy: a renewed commitment to measuring and monitoring vital statistics. Bull World Health Organ 2005; 83:611-7.

26. Núñes MLF, Icaza MGN. Calidad de las estadísticas de mortalidade em Chile, 1997- 2003. Rev Méd Chile 2006; 134:1191-6.

27. Penchansky R, Thomas JW. The concept of access: definition and relationship to consumer satisfaction. Med Care 1981; 19:127-40.

28. Leal MC, Viacava, F. Maternidades do Brasil. RADIS Comunicação em Saúde 2002; (2):8-26.

29. Ministério da Saúde. Saúde da Família no Brasil: uma análise de indicadores selecionados, 19982004. Brasília: Ministério da Saúde; 2006. (Série Projetos, Programas e Relatórios).

30. Frias PG, Vidal SA, Pereira PMH, Lira PIC, Vanderley LC. Avaliação da notificação de óbitos infantis ao Sistema de Informações sobre Mortalidade: um estudo de caso. Rev Bras Saúde Matern Infant 2005; 5 Suppl 1:S43-52.

31. Rede Interagencial de Informação em Saúde. Indicadores básicos para a saúde no Brasil: conceitos e aplicações. 2a Ed. Brasília: Organização Pan-Americana da Saúde; 2008.

Recebido em 14/Mai/2009

Versão final reapresentada em 10/Jan/2010

Aprovado em 01/Mar/2010 